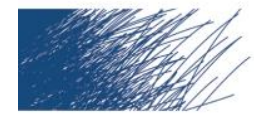

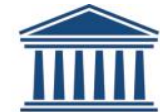 \\ UNIVERSITEIT \\ GENT
}

biblio.ugent.be

The UGent Institutional Repository is the electronic archiving and dissemination platform for all UGent research publications. Ghent University has implemented a mandate stipulating that all academic publications of UGent researchers should be deposited and archived in this repository. Except for items where current copyright restrictions apply, these papers are available in Open Access.

This item is the archived peer-reviewed author-version of: In-Line UV spectroscopy for the quantification of low-dose active ingredients during the manufacturing of pharmaceutical semi-solid and liquid formulations

Authors: Bostijn N., Hellings M., Van der Veen M., Vervaet C., De Beer T.

In: Analytica Chimica Acta, 1013: 54-62

To refer to or to cite this work, please use the citation to the published version:

Bostijn N., Hellings M., Van der Veen M., Vervaet C., De Beer T. (2018) In-Line UV spectroscopy for the quantification of low-dose active ingredients during the manufacturing of pharmaceutical semi-solid and liquid formulations

Analytica Chimica Acta, 1013: 54-62

DOI: 10.1016/j.aca.2018.02.007 


\section{In-line UV spectroscopy for the quantification of low-dose active ingredients during the manufacturing of pharmaceutical semi-solid and liquid formulations}

N. Bostijn ${ }^{a}$, M. Hellings ${ }^{b}, M$. Van Der Veen ${ }^{b}, C$. Vervaet $^{c}, T$. De Beer ${ }^{a},{ }^{*}$.

a Laboratory of Pharmaceutical Process Analytical Technology, Ghent University, Ottergemsesteenweg 460, 9000 Ghent, Belgium

b Johnson \& Johnson Pharmaceutical Research and Development, Analytical Development, Turnhoutseweg 30, 2340 Beerse, Belgium

' Laboratory of Pharmaceutical Technology, Ghent University, Ottergemsesteenweg 460, 9000 Ghent, Belgium

${ }^{*}$ Corresponding author: Thomas De Beer

Ghent University

Laboratory of Pharmaceutical Process Analytical Technology,

Ottergemsesteenweg 460

9000 Ghent (Belgium)

Tel. +32 92648097

Fax +32 92228236

E-mail: Thomas.DeBeer@UGent.be 


\section{Abstract}

2 UltraViolet (UV) spectroscopy was evaluated as an innovative Process Analytical

3 Technology (PAT) - tool for the in-line and real-time quantitative determination of low-

4 dosed active pharmaceutical ingredients (APIs) in a semi-solid (gel) and a liquid

5 (suspension) pharmaceutical formulation during their batch production process. The

6 performance of this new PAT-tool (i.e., UV spectroscopy) was compared with an already

7 more established PAT-method based on Raman spectroscopy. In-line UV measurements

8 were carried out with an immersion probe while for the Raman measurements a non-

9 contact PhAT probe was used. For both studied formulations, an in-line API quantification

10 model was developed and validated per spectroscopic technique. The known API

11 concentrations $(Y)$ were correlated with the corresponding in-line collected preprocessed

12 spectra (X) through a Partial Least Squares (PLS) regression. Each developed

13 quantification method was validated by calculating the accuracy profile on the basis of the

14 validation experiments. Furthermore, the measurement uncertainty was determined

15 based on the data generated for the determination of the accuracy profiles. From the

16 accuracy profile of the UV- and Raman-based quantification method for the gel, it was

17 concluded that at the target API concentration of $2 \%(w / w), 95$ out of 100 future routine

18 measurements given by the Raman method will not deviate more than $10 \%$ (relative error)

19 from the true API concentration, whereas for the UV method the acceptance limits of 10

$20 \%$ were exceeded. For the liquid formulation, the Raman method was not able to quantify

21 the API in the low-dosed suspension (0.09\% (w/w) API). In contrast, the in-line UV method

22 was able to adequately quantify the API in the suspension. This study demonstrated that

23 UV spectroscopy can be adopted as a novel in-line PAT-technique for low-dose 
24 quantification purposes in pharmaceutical processes. Important is that none of the two

25 spectroscopic techniques was superior to the other for both formulations: the Raman

26 method was more accurate in quantifying the API in the gel (2\% (w/w) API), while the UV

27 method performed better for API quantification in the suspension $(0.09 \%(w / w) ~ A P I)$.

28 Keywords

29 In-line UV spectroscopy, In-line Raman spectroscopy, Semi-solids, Liquids, Process

30 Analytical Technology (PAT), Accuracy profile.

\section{1. INTRODUCTION}

32 Spectroscopic techniques are increasingly proposed as alternative methods for the

33 quantification of APls in pharmaceuticals. This is due to their advantages over the

34 traditional techniques, such as fast, in-line, non-invasive and non-destructive

35 measurements without the need of sample preparation. Near infrared (NIR) and Raman

36 spectroscopy have been identified as effective PAT-tools for real-time measurements of

37 critical process and product attributes during pharmaceutical processing. Raman

38 spectroscopy is until now mostly applied for solid dosage forms [1]-[6]. Some in-line

39 quantitative applications for hot-melt extrusion processes have also been reported [7]-[9].

40 Raman spectroscopy has an added value for quantification purposes of pharmaceutical

41 formulations where water is present, such as in semi-solid and liquid formulations, since

42 water produces almost no Raman signal. Research has already been conducted to

43 investigate the opportunity offered by Raman spectroscopy for these formulations [10]-

44 [15], however less frequently as an in-line analytical tool [16]. For some applications, these

45 spectroscopic techniques are not feasible, such as those that require the quantification of 
46 low-dosed analytes. Fluorescence spectroscopy can be an alternative to the conventional

47 spectroscopic techniques for these applications because of its high sensitivity and 48 detection sensitivity [17], [18]. A drawback of fluorescence spectroscopy is that the analyte

49 needs to be a native fluorophore in order to detect it, which limits the number of possible

50 applications for this technique [19].

51 UV spectroscopy is a widely used quantitative analytical technique that finds its 52 application in many research domains and is capable of quantifying very low 53 concentrations (<0.01\%) [20]-[24]. Nevertheless, studies describing on-line and in-line

54 applications of UV/VIS spectroscopy with fibre-optic probes are limited. O'Keeffe et al.

55 monitored the ozone concentration of a gas in an aluminium glass cell with a fibre-based

56 UV/VIS spectroscopy system [25]. Quinn et al. followed the reaction of a nucleoside with

57 trityl chloride in pyridine in a liquid environment [26], using a fibre-optic transmission

58 probe. The concentration of starting material and product was predicted via a PLS

59 regression model. Furthermore, a mixing study using a fibre-optic UV/VIS monitoring

60 technique was reported by $\mathrm{Ng}$ and Assirelli [27]. In this paper, bromophenol blue sodium

61 salt was used as a non-reactive tracer in distilled water. A good agreement between the

62 UV/VIS technique and the traditional conductivity technique was found. Other examples

63 of on-line and in-line UV spectroscopic applications in literature are drug dissolution tests,

64 where the drug release was monitored in real-time [24], [28]. However, the use of UV

65 spectroscopy for in-line monitoring of critical quality attributes during pharmaceutical

66 manufacturing processes of semi-solids and liquids is not yet described in literature.

67 In this study, UV spectroscopy was evaluated as a new PAT-tool for the in-line and real68 time monitoring of the API concentration during the production of pharmaceutical semi- 
69 solid and liquid formulations. Furthermore, the performance of this new PAT-tool was

70 compared with an already established and widely adopted PAT-method based on Raman

71 spectroscopy. The in-line UV spectroscopic measurements were carried out by an

72 immersion probe. For the in-line Raman measurements, a PhAT probe was used. This

73 type of Raman probe was until now only applied in pharmaceutical unit operations such

74 as milling, blending and coating of solid dosage forms [29]. A pharmaceutical gel and

75 suspension with an API concentration of 2 and $0.09 \%(w / w)$, respectively, were selected

76 as model formulations. For both formulations, a PLS regression model was developed per

77 spectroscopic technique and the quantification abilities of both techniques were

78 compared. The validation of the calibration models was assessed via accuracy profiles, a

79 validation strategy for quantitative analytical procedures proposed by the Société

80 Francaise des Sciences et Techniques Pharmaceutiques (SFSTP) [30]-[32].

81 2. MATERIALS AND METHODS

\section{2.1. Materials}

83 Commercially available pharmaceutical formulations were kindly provided by Janssen

84 Pharmaceutica (Beerse, Belgium): a semi-solid (gel) and a liquid (suspension), having an

85 API target concentration of $2 \%$ and $0.09 \%(w / w)$, respectively. Laboratory-scale batches

86 of the formulations were manufactured based on confidential information provided by

87 Janssen Pharmaceutica.

88 2.2. Methods 
90 All formulations were produced with a customized IKA LR2000 mixing system (IKA,

91 Staufen, Germany). The mixing vessel was equipped with a heated jacket for controlling

92 the temperature of the process using a water bath (Type 1032, GFL, Burgwedel,

93 Germany). Interface openings were provided in the cover of the mixing vessel for the

94 implementation of the UV and Raman probe (figure 1).

\section{2.2.2. Calibration and validation samples}

96 In total, one calibration batch and three validation batches were produced for each

97 formulation. Validation batch one and three were produced by operator $\mathrm{A}$ and validation

98 batch two by operator $\mathrm{B}$. Also, the validation batches were produced on three different

99 days. Instead of producing a complete batch for each concentration level of the calibration

$100(80,90,95,100,105,110$ and $120 \%$ relative to target) and validation $(85,95,100,105$

101 and $115 \%$ relative to target) set, all the concentration levels were created using one

102 calibration batch and three validation batches (three different days). This was done by the

103 stepwise addition of API to a batch, corresponding to the different concentration levels.

104 The calibration batch was produced following the standard batch production procedure of

105 the formulations. However, instead of producing a batch with the target API concentration

106 (i.e., $100 \%$ of target), the calibration batch contained only $80 \%$ of the target API

107 concentration. After completing batch manufacturing, spectra of the lowest concentration

108 level (i.e., $80 \%$ of target) were collected in-line while the formulation was being mixed.

109 Next, a specific amount of API was added to the calibration batch, corresponding to the 110 subsequent concentration level (i.e., $90 \%$ of target), followed by the collection of spectra.

111 These steps (i.e., API addition and spectra recording) were repeated until the highest 112 concentration level (i.e., $120 \%$ of target) was reached for the calibration batch, and 
113 spectra were recorded at each concentration. The validation batches were produced

114 following the same procedure as described for the calibration batch, but with other

115 concentration levels $(85,95,100,105$ and $115 \%$ relative to target). During this procedure

116 (i.e., API addition and spectra recording), the formulation was mixed with a constant 117 mixing speed.

\section{2.2.3. UV spectroscopy}

119 An Avaspec-ULS2048L spectrometer (Avantes, Apeldoorn, The Netherlands), equipped

120 with a CCD detector, was connected by a fibre-optic cable to an immersion probe with a

12145 degree angle window. The probe contained six illumination fibres and one detection

122 fibre. The light source was an AvaLight Deuterium-Halogen Lamp. All spectra were

123 acquired in the $200-1100 \mathrm{~nm}$ spectral range. The exposure time was $1000 \mathrm{~ms}$ and 950

$124 \mathrm{~ms}$ for the gel and suspension, respectively, with each spectrum the average of 5 scans

125 and a total of 40 spectra/concentration level. The immersion probe was inserted via the 126 cover of the mixing vessel through a custom made interface (figure 1b).

\section{2.2.4. Raman spectroscopy}

128 In-line Raman spectra were recorded using a Raman Rxn2 spectrometer (Kaiser Optical

129 Systems, Ann Arbor, MI, USA), equipped with a CCD detector and a fibre-optic PhAT

130 probe. The laser wavelength was $785 \mathrm{~nm}$ with a laser power of $400 \mathrm{~mW}$. The spectral

131 range of the system was $150-1890 \mathrm{~cm}^{-1}$ with a resolution of $5 \mathrm{~cm}^{-1}$. For all formulations

132 an exposure time of $15 \mathrm{~s}$ with no averaging was used and every $30 \mathrm{~s}$ a spectrum was

133 recorded. Per concentration level, 30 spectra were collected in-line for both the calibration

134 and validation sets. The Raman PhAT probe was implemented through an opening in the 
135 cover of the mixing vessel and fixed with a sealing to ensure a fixed probe position (figure 136 1c).

137 2.2.5. Development of the calibration models

138 For each formulation one calibration model per spectroscopic technique was developed

139 (table 1). The UV calibration model of the gel was developed applying mean-centering,

140 Standard Normal Variate (SNV) correction and first-derivative transformation as

141 preprocessing methods in combination with selecting the spectral region between 280 -

$142297 \mathrm{~nm}$ (table 1). The Raman spectra of the gel were mean-centered and SNV corrected,

143 followed by taking the first derivative and selecting the spectral regions where the API

144 showed Raman activity. SNV preprocessing was applied to eliminate baseline offset

145 variations, which can be caused by scatter differences between the samples. First

146 derivative transformation allowed a better visualization of small absorption bands and

147 corrected for baseline shifts [33].

148 The API concentrations $(\mathrm{Y})$ were regressed against the corresponding in-line collected 149 preprocessed spectra (X) through a PLS method. The goodness of fit and the predictive 150 ability of the developed PLS models were assessed by the calculation of $R^{2}$ and $Q^{2}$, 151 respectively. $\mathrm{Q}^{2}$ values were obtained after performing a leave-one-out cross-validation,

152 in which sub-models were developed from a reduced calibration dataset and the excluded 153 data was predicted by the sub-models. The number of PLS components providing the 154 highest $\mathrm{Q}^{2}$ value was selected. Details of the developed UV and Raman PLS models of 155 the suspension are also displayed in table 1. The PLS models were created using the 156 SIMCA software (Version 14, Umetrics, Umeå, Sweden). 


\subsubsection{Validation of the calibration models}

158 The predictive properties of the developed models were first assessed by computation of

159 the Root Mean Square Error of Prediction (RMSEP), obtained when predicting the

160 external validation sets. During validation, the within-day, between-day and operator

161 variability were incorporated. Accuracy profiles were adopted to evaluate the validation of

162 the developed analytical methods and are proposed by SFSTP as a harmonized approach

163 for the validation of quantitative analytical procedures [30]-[32]. The objective of validation

164 is to ensure that the difference between the measured value $\left(x_{i}\right)$ and the unknown true

165 value of the sample $(\mu \mathrm{T})$ will be lower than an acceptance limit $(\lambda)$ :

166

$$
\left|x_{i}-\mu_{T}\right|<\lambda
$$

167 Here, $\lambda$ was set at $10 \%$. For an analytical method to be considered as acceptable, it must

168 be assured that the probability that a measurement will fall outside the acceptance limits 169 is less than or equal to the maximum risk that the analyst is able to take during routine 170 use:

$$
\operatorname{Pr}\left(\left|x_{i}-\mu_{T}\right|<\lambda\right) \geq \beta
$$

172 The desired proportion of measurements inside the acceptance limits $(\beta)$ was set at 95

$173 \%$. The computation of a large number of validation parameters (e.g., precision, trueness,

174 linearity, ...) is not sufficient to decide whether the objectives of validation are ensured.

175 Therefore, the accuracy profile was used as a decision tool for the validity of the analytical

176 methods, which is constructed from the total error of the method, being the sum of the

177 random error (precision) and systematic error (trueness) [32]. For the precision, both the 
178 repeatability (within-day variability) and intermediate precision (between-day and operator

179 variability) were calculated [34]. In the accuracy profiles, the acceptance limits are plotted

180 together with the relative error of the individual predictions, the relative bias and the $\beta$ -

181 expectation tolerance intervals at each concentration level of the validation set. Here, the

182 acceptance limits were set at $10 \%$ relative error. The $\beta$-expectation tolerance intervals

183 visualise at each concentration level where at least 95 out of 100 future measurements

184 given by the analytical procedure will fall between [35]. The intersect between the

185 acceptance limits and the $\beta$-expectation tolerance intervals defines the upper and lower

186 quantification limits of the analytical method. The accuracy profiles were calculated from

187 the data obtained from the validation experiments.

188 Furthermore, the standard deviation of the $\beta$-expectation tolerance intervals was used for

189 the estimation of the standard uncertainty in the measurements [36]. The uncertainty is 190 defined as a parameter associated with the result of a measurement, that characterises

191 the dispersion of the values that could reasonably be attributed to the measurand. The

192 measurement uncertainty was expressed by four uncertainty parameters: uncertainty of

193 the bias, uncertainty (combination of uncertainty of the bias with the intermediate precision

194 standard deviation), expanded uncertainty and the relative expanded uncertainty. The 195 expanded uncertainty represents an interval around the mean value where the unknown

196 true value can be located with a certain confidence level (here $95 \%$ ). The relative 197 expanded uncertainty is calculated as the expanded uncertainty divided by the 198 corresponding true concentration [37].

\section{3. RESULTS AND DISCUSSION}


200 The development and validation of the PLS models for the gel formulation, based on the

201 measurements with the two spectroscopic techniques (UV and Raman spectroscopy), will

202 be discussed in detail in the results section. Information regarding the development and

203 validation of the PLS models of the suspension can be found in tables 1, 2, 3 and 4.

204 3.1. Development of the calibration models

205 3.1.1. UV spectroscopy

206 The in-line UV/VIS measurements were made in the $200-1100 \mathrm{~nm}$ spectral range. Only

207 the UV region (200 - $400 \mathrm{~nm}$ ) was investigated, since the size of the conjugated system

208 of the API was not large enough to absorb in the VIS region [38]. Also, prominent

209 deuterium peaks were present in the VIS region (486 and $656 \mathrm{~nm}$ ), which were not of

210 interest [39]. In a first step, the molecular structure of the API in the gel was screened for

211 UV activity. Several aromatic groups were found in the molecular structure and suggested

212 that the API will absorb in the UV region. The exact absorption wavelength is dependent

213 of the type and number of functional groups coupled to the aromatic rings, which can shift

214 the absorption wavelength to lower or higher wavelengths [38]. To confirm whether the

215 API could indeed be detected in the UV spectra of the gel, where possible interfering 216 components are present, the spectra of the calibration batch were coloured according to

217 concentration level and it was checked whether the colours were in sequence with the

218 concentration levels. A distinctive peak in the region $280-297 \mathrm{~nm}$ was observed in the

219 SNV-corrected and first-derived UV spectra of the gel, where the spectra were clearly

220 clustered according to API concentration (figure 2). 
221 A PLS model was developed from the mean-centered, SNV-corrected and first-derived

222 UV spectra of the gel between $280-297 \mathrm{~nm}\left(R^{2}=0.988 ; Q^{2}=0.988 ;\right.$ Root Mean Square

223 Error of Cross Validation $($ RMSECV) $=0.0274 \% \mathrm{w} / \mathrm{w}$ ) (table 1 and 2 ). Selecting this

224 spectral region eliminated interfering variance sources, thereby increasing the variance 225 due to concentration differences. RMSEP values $(0.0584,0.0709$ and $0.0588 \% \mathrm{w} / \mathrm{w})$ of

226 the gel were calculated from the predictions of the three validation batches. Also for the

227 suspension a calibration model was developed, following the same strategy as described

228 for the gel (table 1 and 2).

\section{3.1.2. Raman spectroscopy}

230 The Raman spectra of the gel formulation (calibration batch) and pure API are presented

231 in figure 3 . The peaks in the spectra of the pure API with the highest intensity are situated

232 around $396,660,1348$ and $1590 \mathrm{~cm}^{-1}$. It can be noticed from figure 3 that at these Raman

233 shifts, peaks in the spectra of the gel are visible. A detail of the preprocessed spectra of

234 the gel calibration set at the above mentioned spectral regions is shown in figure 4.

235 Applying these preprocessing methods highlighted the spectral differences most. A logic

236 concentration trend in the spectra was observed at the API selective bands: increasing

237 Raman intensity for an increasing API concentration. These four regions were the most

238 abundant peaks in the Raman spectra of the pure API (figure 3), suggesting that the trend

239 in the spectra was caused by the difference in API concentration.

240 The model of the gel formulation with the highest predictive performance $\left(R^{2}=0.973 ; Q^{2}\right.$

$241=0.973 ; \mathrm{RMSECV}=0.0418 \% \mathrm{w} / \mathrm{w}$ ) was created from the mean-centered, SNV corrected 242 and first-derived Raman spectra in the regions $390-405,655-667,1340-1355$ and 
$2431570-1600 \mathrm{~cm}^{-1}$ (table 1). The selection of these spectral regions was based on the

244 evaluation of the Raman spectra of the pure API and gel (figure 3 and 4). The resulting

245 RMSEP values of the three validation sets were $0.0255,0.0235$ and $0.0381 \%(w / w)$. The

246 PLS model of the suspension, measured with the Raman PhAT probe, was constructed

247 using the same strategy as described above and detailed information regarding the

248 construction of the model together with the resulting RMSECV and RMSEP values can

249 be found in table 1 and 2 .

250

251

252

\subsection{Validation of the calibration models}

\subsubsection{UV spectroscopy}

253 The accuracy profile for the UV-based in-line quantification method of the gel is displayed

254 in figure 5a. At each validation concentration level, the $\beta$-expectation tolerance intervals 255 exceeded the acceptance limits (10\% relative error) (figure 5a). Furthermore, the

256 predictions of the lowest API concentration level $(1.75 \% \mathrm{w} / \mathrm{w})$ were more biased than the

257 other concentration levels (table 3 ). This is probably because of the difficulty to detect this

258 low API concentration. The calculated precision parameters (repeatability and

259 intermediate precision) from the UV-based in-line quantification method showed that the

260 intermediate precision Relative Standard Deviation (RSD) was much higher compared to

261 the repeatability RSD at all concentration levels (table 3). Because of the lower

262 intermediate precision, an important day or operator effect was causing variability in the

263 predictions.

264 The accuracy profile of the UV-based in-line quantification method of the suspension is 265 displayed in figure 6a. Between the API concentration range of $0.0865-0.0955 \%(w / w)$, 
266 the $\beta$-expectation tolerance intervals fell within the acceptance limits of $10 \%$ (relative

267 error). Therefore, future measurements between an API concentration of 0.0865 and

$2680.0955 \%(\mathrm{w} / \mathrm{w})$ obtained by this procedure have a probability of $95 \%$ that the difference

269 between the measured concentration and the true concentration is less than $10 \%$ (relative

270 error). However, the $\beta$-expectation tolerance intervals at the lowest $(0.0774 \% \mathrm{w} / \mathrm{w})$ and

271 highest $(0.1046 \% \mathrm{w} / \mathrm{w})$ API concentration level were almost exceeding the $20 \%$ (relative

272 error) acceptance limits. The relative bias at API concentration level 0.0774 and 0.1046

$273 \%(\mathrm{w} / \mathrm{w})$ was 3.04 and $-4.05 \%$, respectively. This value is remarkably higher than the

274 relative bias $(1.40,0.65$ and $-0.92 \%)$ of the other validation concentration levels.

275 Furthermore, a higher imprecision for the lowest and highest concentration level was

276 observed, which was mainly induced by a low intermediate precision, suggesting an

277 important day or operator effect. Table 2 shows that the RMSEP of day $1(0.00496 \% \mathrm{w} / \mathrm{w})$

278 was almost four times higher than the RMSEP of day $2(0.00148 \% \mathrm{w} / \mathrm{w})$ and $3(0.00171$

$279 \% \mathrm{w} / \mathrm{w})$. A cause for the less accurate predictions of the day 1 validation samples was not

280 found, but could be operator related such as an accidental alteration in the production

281 process of these validation samples.

\section{3.2.2. Raman spectroscopy}

283 For the accuracy profile of the Raman-based in-line quantification method of the gel, the

$284 \beta$-expectation tolerance intervals exceeded the $10 \%$ (relative error) acceptance limits only

285 at the $1.75 \%(w / w)$ API concentration level (figure 5b). Hence, in the $1.96-2.37 \%(w / w)$

286 API concentration range, 95 out of 100 future measurements will be included within the

287 acceptance limits of $10 \%$ (relative error) and even within the $5 \%$ (relative error)

288 acceptance limits, when using this analytical method. To explain the large $\beta$-expectation 
289 tolerance interval at the $1.75 \%(\mathrm{w} / \mathrm{w})$ API concentration level, the trueness and precision

290 were investigated. The calculated relative bias and RSD for repeatability at this level were

291 not higher than for the other concentration levels, but the intermediate precision RSD was

292 higher (table 3). There was indeed one validation batch (day 3) where the predictions of

293 the lowest concentration level were lower in comparison to the other validation batches.

294 This variability could be caused by the detection sensitivity limitations of the Raman

295 method at the lowest concentration level.

296 The accuracy profile for the in-line Raman-based quantification method of the suspension

297 was developed following the same strategy as described above and is displayed in figure

298 6b. The $\beta$-expectation tolerance intervals exceeded the $10 \%$ (relative error) acceptance

299 limits over the whole concentration range, except for the API concentration levels 0.0862

300 and $0.0953 \%(\mathrm{w} / \mathrm{w})$. The accuracy profile has a clear downward trend, i.e., low

301 concentration levels were predicted higher, the intermediate concentration level was

302 predicted around the target concentration and the high concentration levels were

303 predicted lower. This demonstrated that all the concentration levels were predicted as the

304 same value, indicating that the small changes in API concentration could not be detected

305 and that the quantification of the low-dosed API in this suspension could not be achieved

306 with Raman spectroscopy.

307 When the accuracy profiles and validation parameters of the UV and Raman quantification

308 methods of the suspension are compared, it is clear that the in-line quantification of the

309 API only was possible with UV spectroscopy (table 2 and 3). To better understand the

310 difference in predictive performance of both spectroscopic techniques, the in-line UV and

311 Raman spectra of the suspension calibration set were investigated (figure 7). The UV 
312 spectra are clearly separated according to API concentration between $310-325 \mathrm{~nm}$,

313 which confirmed the quantification ability and high sensitivity of UV spectroscopy for this

314 API. In the Raman spectra, no spectral differences between the concentration levels are

315 seen and no API specific peaks can be located in the spectra of the suspension, despite

316 investigating a region of the spectra where the API is Raman active. Increasing the

317 exposure time and number of scans of the Raman spectrometer had no impact on the 318 detection of the API.

319 The high sensitivity of UV spectroscopy was correlated with the strong UV activity of the

$320 \mathrm{API}$ in the suspension, due to conjugated double bonds in its molecular structure [38],

321 [40]. However, the molecular structure of the API also meets to the requirements (non-

322 polar bonds and aromatic rings) for good Raman activity, suggesting that the failure of the

323 Raman method for the suspension is linked to the inherent weak Raman effect [17], [41].

324 Raman spectroscopy applies monochromatic light to irradiate the samples and the

325 incident light is scattered by the sample molecules. Most of this light is scattered at the

326 same frequency, i.e., Raleigh radiation. Only one in $10^{8}$ incident photons is scattered with

327 a different frequency than the incident light (Raman effect). This in combination with the

328 small fraction of light which is scattered into the same direction of the probe, explains why

329 the quantification of low concentrations can be an issue for Raman spectroscopy [41].

330 UV spectroscopy was identified as a novel and alternative in-line spectroscopic tool for

331 quantification purposes, in addition to the widely used Raman spectroscopy. Important is

332 that none of the two spectroscopic techniques was superior to the other for both the

333 formulations. While Raman was more accurate in quantifying the API in the gel ( $2 \% \mathrm{w} / \mathrm{w})$,

334 the in-line UV-based method for the suspension performed better than the in-line Raman- 
335 based method. This study illustrated that spectroscopic techniques can be complementary

336 and that the preferred technique is dependent on several factors such as the molecular

337 structure of the API, concentration of the analyte, measurement conditions, presence of

338 interfering components, measurement time and cost. In addition, the UV immersion probe

339 was more practical to work with inside a process environment, because the probe tip can

340 be in direct contact with the sample. Furthermore, UV spectroscopy is a suitable PAT-tool

341 for measurements in aqueous environments, since the suspension contained water. This

342 would be challenging for NIR spectroscopy because water creates strong absorbance

343 peaks in the near infrared region, which can potentially overwhelm the signal(s) of the API

344 [41]. Preliminary off-line experiments with NIR spectroscopy showed that the APIs had

345 weak signals in the near infrared region and therefore NIR spectroscopy was not further

346 investigated in this study.

347 The measurement uncertainty of the UV- and Raman-based calibration models is 348 summarized in table 4 in terms of the uncertainty of the bias, uncertainty, expanded 349 uncertainty and the relative expanded uncertainty at each concentration level of the 350 validation sets [36]. For the UV-based method of the suspension, the relative expanded 351 uncertainty at the target API concentration $(0.09 \% \mathrm{w} / \mathrm{w})$ was $3.82 \%$ (relative error) (table 352 4). This means that the unknown true value is located at a maximum of $\pm 3.82 \%$ (relative

353 error) around the measured value, with a confidence level of $95 \%$. In comparison, the 354 relative expanded uncertainty at the target concentration of the suspension was $6.53 \%$ 355 (relative error) for the Raman-based method.

\section{4. CONCLUSIONS}


357 In this study, analytical methods based on in-line UV spectroscopy were developed for

358 the quantification of APIs in pharmaceutical semi-solid and liquid formulations. The 359 performance of this new PAT-tool was compared with an already more established PAT-

360 method based on Raman spectroscopy. In-line UV measurements were carried out with

361 an immersion probe while for the Raman measurements a PhAT probe was used. The

362 validation of the analytical methods was evaluated by the calculation of accuracy profiles,

363 ensuring that 95 out of 100 future routine measurements will be included within the present

364 acceptance limits of $10 \%$ (relative error). Furthermore, the uncertainty of bias and the

365 expanded uncertainty were estimated at each concentration level. The results show that

366 the calibration model developed from the Raman PhAT probe data had a higher accuracy

367 than the UV-based model for the gel formulation (2\% (w/w) API). The UV method

368 developed for the low-dosed suspension (0.09\% (w/w) API) had good performance

369 characteristics, whereas the quantification of this low concentration was not possible with

370 Raman spectroscopy due to detection sensitivity limitations. It was demonstrated that UV

371 spectroscopy can be adopted as a novel PAT-tool for in-line and real-time quantification

372 purposes during the manufacturing of pharmaceutical semi-solid and liquid formulations

373 and that it can be complementary to other spectroscopic techniques, especially when the

374 detection sensitivity is not sufficient. However, the feasibility of the spectroscopic

375 technique is case dependent and should therefore be assessed in preliminary feasibility 376 studies.

377 FUNDING

378 Research funded by a PhD grant of the Research Foundation Flanders (FWO). 


\section{REFERENCES}

[1] Y. Roggo, K. Degardin, and P. Margot, "Identification of pharmaceutical tablets by Raman spectroscopy and chemometrics," Talanta, vol. 81, no. 3, pp. 988-995, 2010.

[2] S. Romero-Torres, J. D. Pérez-Ramos, K. R. Morris, and E. R. Grant, "Raman spectroscopic measurement of tablet-to-tablet coating variability," J. Pharm. Biomed. Anal., vol. 38, no. 2, pp. 270-274, 2005.

[3] T. R. M. De Beer, C. Bodson, B. Dejaegher, B. Walczak, P. Vercruysse, A. Burggraeve, A. Lemos, L. Delattre, Y. Vander Heyden, J. P. Remon, C. Vervaet, and W. R. G. Baeyens, "Raman spectroscopy as a process analytical technology (PAT) tool for the in-line monitoring and understanding of a powder blending process," J. Pharm. Biomed. Anal., vol. 48, no. 3, pp. 772-779, 2008.

[4] D. S. Hausman, R. T. Cambron, and A. Sakr, "Application of Raman spectroscopy for on-line monitoring of low dose blend uniformity," Int. J. Pharm., vol. 298, no. 1, pp. 80-90, 2005.

[5] I. Lewis and L. S. Taylor, "spectroscopic techniques Comparison of Sampling Techniques for In-Line Monitoring Using Raman Spectroscopy," Appl. Spectrosc., vol. 59, no. 7, 2005.

[6] G. J. Vergote, T. R. M. De Beer, C. Vervaet, J. P. Remon, W. R. G. Baeyens, N. Diericx, and F. Verpoort, "In-line monitoring of a pharmaceutical blending process using FT-Raman spectroscopy," Eur. J. Pharm. Sci., vol. 21, no. 4, pp. 479-485, 2004.

[7] L. Saerens, L. Dierickx, B. Lenain, C. Vervaet, J. P. Remon, and T. De Beer, "Raman spectroscopy for the in-line polymer-drug quantification and solid state characterization during a pharmaceutical hot-melt extrusion process," Eur. J. Pharm. Biopharm., vol. 77, no. 1, pp. 158-163, 2011.

[8] P. D. Coates, S. E. Barnes, M. G. Sibley, E. C. Brown, H. G. M. Edwards, and I. J. Scowen, "In-process vibrational spectroscopy and ultrasound measurements in polymer melt extrusion," Polymer (Guildf)., vol. 44, no. 19, pp. 5937-5949, 2003.

[9] S. Barnes and M. Sibley, "Process monitoring of polymer melts using in-line spectroscopy," Trans. Inst. Meas. Control, vol. 5, pp. 453465, 2007.

[10] T. R. M. De Beer, W. R. G. Baeyens, A. Vermeire, D. Broes, J. P. Remon, and C. Vervaet, "Raman spectroscopic method for the determination of medroxyprogesterone acetate in a pharmaceutical suspension: validation of quantifying abilities, uncertainty assessment and comparison with the high performance liquid chromatography reference method," Anal. Chim. Acta, vol. 589, no. 2 pp. 192-199, 2007.

[11] S. C. Park, M. Kim, J. Noh, H. Chung, Y. Woo, J. Lee, and M. S. Kemper, "Reliable and fast quantitative analysis of active ingredient in pharmaceutical suspension using Raman spectroscopy," Anal. Chim. Acta, vol. 593, no. 1, pp. 46-53, 2007.

[12] M. Kim, H. Chung, Y. Woo, and M. S. Kemper, "A new non-invasive, quantitative Raman technique for the determination of an active ingredient in pharmaceutical liquids by direct measurement through a plastic bottle," Anal. Chim. Acta, vol. 587, no. 2, pp. 200-207, 2007.

[13] B. Gotter, W. Faubel, S. Heißler, J. Hein, and R. Neubert, "Determination of drug content in semisolid formulations by non-invasive spectroscopic methods: FTIR-ATR, -PAS, -Raman and PDS," J. Phys. Conf. Ser., vol. 214, p. 12129, 2010.

[14] T. R. M. De Beer, W. R. G. Baeyens, Y. Vander Heyden, J. P. Remon, C. Vervaet, and F. Verpoort, "Influence of particle size on the quantitative determination of salicylic acid in a pharmaceutical ointment using FT-Raman spectroscopy," Eur. J. Pharm. Sci., vol. 30, no. 3-4, pp. 229-235, 2007.

[15] M. T. Islam, Rodriguez-Hornedo, S. Ciotti, and C. Ackermann, "The potential of Raman spectroscopy as a process analytical technique during formulations of topical gels and emulsions," Pharm. Res., vol. 21, no. 10, pp. 1844-1851, 2004.

[16] T. R. M. De Beer, W. R. G. Baeyens, J. Ouyang, C. Vervaet, and J. P. Remon, "Raman spectroscopy as a process analytical technology tool for the understanding and the quantitative in-line monitoring of the homogenization process of a pharmaceutical suspension," $R$. Soc. Chem., vol. 131, pp. 1137-1144, 2006.

[17] K. A. Bakeev, Process Analytical Technology - Second Edition. 2010.

[18] S. Warnecke, A. Rinnan, M. Allesø, and S. B. Engelsen, "Fluorescence Spectroscopy in Process Analytical Technology ( PAT ): Simultaneous Quantification of Two Active Pharmaceutical Ingredients in a Tablet Formulation," Appl. Spectrosc., vol. 69, no. 3, pp. 323-331, 2015.

[19] N. Shanker and S. L. Bane, "Basic Aspects of Absorption and Fluorescence Spectroscopy and Resonance Energy Transfer Methods," Methods Cell Biol., vol. 84, no. 7, pp. 213-242, 2008.

[20] J. J. Moes, M. M. Ruijken, E. Gout, H. W. Frijlink, and M. I. Ugwoke, "Application of process analytical technology in tablet process development using NIR spectroscopy: Blend uniformity, content uniformity and coating thickness measurements," Int. J. Pharm., vol. 357, no. 1-2, pp. 108-118, 2008.

[21] G. M. Hadad, A. El-Gindy, and W. M. M. Mahmoud, "HPLC and chemometrics-assisted UV-spectroscopy methods for the simultaneous determination of ambroxol and doxycycline in capsule," Spectrochim. Acta - Part A Mol. Biomol. Spectrosc., vol. 70, no. 3, pp. 655-663, 2008.

[22] D. Bonazzi, R. Gotti, V. Andrisano, and V. Cavrini, "Analysis of ACE inhibitors in pharmaceutical dosage forms by derivative UV spectroscopy and liquid chromatography (HPLC)," J. Pharm. Biomed. Anal., vol. 16, no. 3, pp. 431-438, 1997.

[23] M. Sumithra, P. Shanmugasundaram, and K. Srinivasulu, "Analytical method development and validation of lafutidine in tablet dosage form by RP-HPLC.," Int. J. ChemTech Res., vol. 3, no. 3, pp. 1403-1407, 2011.

[24] K. Nie, L. Li, X. Li, Y. Zhang, X. Mu, and J. Chen, “Sustained-Release Tablets Release by Test System," Dissolution Technol., no. February, pp. 16-19, 2009.

[25] S. O. Keeffe, C. Fitzpatrick, and E. Lewis, "An optical fibre based ultra violet and visible absorption spectroscopy system for ozone concentration monitoring," Sensors and Actuators, vol. 125, pp. 372-378, 2007.

[26] A. C. Quinn, P. J. Gemperline, B. Baker, M. Zhu, and D. S. Walker, "Fiber-optic UV/visible composition monitoring for process control of batch reactions," Chemom. Intell. Lab. Syst., vol. 45, pp. 199-214, 1999.

[27] D. J. W. Ng and M. Ã. Assirelli, "MIXING STUDY IN BATCH STIRRED VESSELS USING A FIBRE-OPTIC UV-VIS MONITORING TECHNIQUE," 
Chem. Eng. Res. Des., vol. 85, no. 1995, pp. 1348-1354, 2007.

[28] X. Lu, R. Lozano, and P. Shah, "In-Situ Dissolution Testing Using Different UV Fiber Optic Probes and Instruments," Dissolution Technol., pp. 6-15, 2003.

[29] J. Müller, K. Knop, J. Thies, C. Uerpmann, and P. Kleinebudde, "Feasibility of Raman spectroscopy as PAT tool in active coating.," Drug Dev. Ind. Pharm., vol. 36, no. 2, pp. 234-243, 2010.

[30] P. Hubert, J. J. Nguyen-Huu, B. Boulanger, E. Chapuzet, P. Chiap, N. Cohen, P. A. Compagnon, W. Dewé, M. Feinberg, M. Lallier, M. Laurentie, N. Mercier, G. Muzard, C. Nivet, and L. Valat, "Harmonization of strategies for the validation of quantitative analytical procedures A SFSTP proposal-part I," J. Pharm. Biomed. Anal., vol. 36, no. 3, pp. 579-586, 2004.

[31] N. C. Ph. Hubert a, J.-J. Nguyen-Huub, B. Boulanger c, E. Chapuzet d, P. Chiap e, M. L. k P.-A. Compagnong, W. Dewé, M. Feinberg i, M. Lallier j, and E. R. a N. Mercier d, G. Muzardl, C. Nivetm, L. Valat n, "Harmonization of strategies for the validation of quantitative analytical procedures A SFSTP proposal - Part II," J. Pharm. Biomed. Anal., vol. 45, pp. 70-81, 2007.

[32] P.-A. C. Ph. Hubert a, J.-J. Nguyen-Huub, B. Boulanger c, E. Chapuzet d, N. Cohene and E. R. a W. Dew'e g, M. Feinberg h, M. Laurentie i, N. Mercier d, G. Muzardj, L. Valat k, "Harmonization of strategies for the validation of quantitative analytical procedures A SFSTP proposal-Part III," J. Pharm. Biomed. Anal., vol. 45, pp. 82-96, 2007.

[33] J. Luypaert, S. Heuerding, Y. Vander Heyden, and D. L. Massart, "The effect of preprocessing methods in reducing interfering variability from near-infrared measurements of creams," J. Pharm. Biomed. Anal., vol. 36, pp. 495-503, 2004.

[34] International Conference on Harmonisation of Technical Requirements for Registration of Pharmaceuticals for Human use, "VALIDATION OF ANALYTICAL PROCEDURES: TEXT AND METHODOLOGY Q2 (R1)," 2015.

[35] R. Mee, " $\beta$-Expectation and $\beta$-Content Tolerance Limits for Balanced One-Way ANOVA Random Model," Technometrics, vol. 26, no. 3, pp. 251-254, 1984.

[36] M. Feinberg, B. Boulanger, W. Dewé, and P. Hubert, "New advances in method validation and measurement uncertainty aimed at improving the quality of chemical data," Anal. Bioanal. Chem., vol. 380, no. 3 SPEC.ISS., pp. 502-514, 2004.

[37] Eurachem/CITAC Guide CG 4, Quantifying Uncertainty in Analytical Measurement Third Edition. 2012.

[38] D. G. Watson, Pharmaceutical analysis: a textbook for pharmacy students and pharmaceutical chemists. 1999.

[39] B. M. Ham and A. MaHam, Analytical Chemistry: A Chemist and Laboratory Technician's Toolkit. 2015.

[40] R. M. Silverstein, Spectrometric Identification of Organic Compounds, 6th ed. New York: John Wiley \& Sons, 1998.

[41] T. De Beer, A. Burggraeve, M. Fonteyne, L. Saerens, J. P. Remon, and C. Vervaet, "Near infrared and Raman spectroscopy for the inprocess monitoring of pharmaceutical production processes," Int. J. Pharm., vol. 417, no. 1-2, pp. 32-47, 2011. 


\begin{tabular}{|c|c|c|c|c|}
\hline & \multicolumn{2}{|c|}{ Gel $(2 \% w / w)$} & \multicolumn{2}{|c|}{ Suspension $(0.09 \%$ w/w) } \\
\hline & UV & Raman & UV & Raman \\
\hline Exposure time (s) & 1 & 15 & 0.95 & 15 \\
\hline Scans & 5 & 1 & 5 & 1 \\
\hline $\begin{array}{l}\text { Preprocessing } \\
\text { methods }\end{array}$ & $\begin{array}{c}\text { Mean-centering } \\
\text { SNV } \\
1^{\text {st }} \text { derivative }\end{array}$ & $\begin{array}{c}\text { Mean-centering } \\
\text { SNV } \\
1^{\text {st }} \text { derivative }\end{array}$ & $\begin{array}{l}\text { Mean-centering } \\
1^{\text {st }} \text { derivative }\end{array}$ & $\begin{array}{c}\text { Mean-centering } \\
\text { SNV } \\
1^{\text {st }} \text { derivative }\end{array}$ \\
\hline $\begin{array}{l}\text { Spectral region } \\
\text { (UV: nm, Raman: } \\
\left.\mathrm{cm}^{-1}\right)\end{array}$ & 280.1-296.9 & $\begin{array}{c}390.1-404.8 \\
655.0-666.7 \\
1340.2-1354.9 \\
1570.0-1600.0\end{array}$ & $310.1-325.6$ & $1390.0-1430.2$ \\
\hline $\mathrm{R}^{2}$ & 0.988 & 0.973 & 0.995 & 0.115 \\
\hline$Q^{2}$ & 0.988 & 0.973 & 0.995 & 0.028 \\
\hline $\begin{array}{l}\text { \# of PLS } \\
\text { components }\end{array}$ & 2 & 1 & 2 & 1 \\
\hline
\end{tabular}

Table 1. Exposure time, number of scans, preprocessing methods, spectral region(s), $R^{2}$, $\mathrm{Q}^{2}$ and number of PLS components of the developed calibration models. 


\begin{tabular}{|l|c|c|c|c|}
\hline \multirow{2}{*}{} & \multicolumn{2}{|c|}{ Gel (2 \% w/w) } & \multicolumn{2}{c|}{ Suspension (0.09 \% w/w) } \\
\cline { 2 - 5 } & UV & Raman & UV & Raman \\
\hline RMSECV $(\% \mathrm{w} / \mathrm{w})$ & 0.0274 & 0.0418 & 0.000819 & 0.0108 \\
\hline RMSEP day 1 $\% \mathrm{w} / \mathrm{w})$ & 0.0584 & 0.0255 & 0.00496 & 0.00947 \\
\hline RMSEP day 2 $(\% \mathrm{w} / \mathrm{w})$ & 0.0709 & 0.0235 & 0.00148 & 0.00996 \\
\hline RMSEP day 3 $(\% \mathrm{w} / \mathrm{w})$ & 0.0588 & 0.0381 & 0.00171 & 0.00951 \\
\hline
\end{tabular}

Table 2. RMSECV and RMSEP values of the UV and Raman calibration models for each formulation. 


\begin{tabular}{|c|c|c|c|c|c|}
\hline $\begin{array}{l}\text { Spectroscopic } \\
\text { technique }\end{array}$ & $\begin{array}{l}\text { Concentration } \\
\text { level }(\% \mathrm{w} / \mathrm{w})\end{array}$ & $\begin{array}{c}\text { Relative bias } \\
\text { (\%) }\end{array}$ & $\begin{array}{l}\text { Repeatability } \\
\text { (RSD, \%) }\end{array}$ & $\begin{array}{l}\text { Intermediate } \\
\text { precision } \\
\text { (RSD, \%) }\end{array}$ & $\begin{array}{c}\text { Relative B- } \\
\text { expectation } \\
\text { tolerance limits } \\
(\%) \\
\end{array}$ \\
\hline \multicolumn{6}{|c|}{ Gel $(2 \% w / w)$} \\
\hline \multirow{5}{*}{ UV } & 1.75 & -3.02 & 1.239 & 2.341 & $-11.14 ; 5.10$ \\
\hline & 1.96 & -0.88 & 0.838 & 2.652 & $-13.82 ; 12.05$ \\
\hline & 2.06 & -0.01 & 0.496 & 2.465 & $-12.21 ; 12.19$ \\
\hline & 2.16 & 0.12 & 1.869 & 3.277 & $-11.56 ; 11.80$ \\
\hline & 2.37 & -1.05 & 1.040 & 2.226 & $-11.77 ; 9.66$ \\
\hline \multirow{5}{*}{ Raman } & 1.75 & 0.17 & 0.815 & 2.097 & $-10.12 ; 10.46$ \\
\hline & 1.96 & -0.48 & 1.127 & 1.225 & $-3.46 ; 2.49$ \\
\hline & 2.06 & -1.13 & 0.881 & 0.944 & $-3.36 ; 1.10$ \\
\hline & 2.16 & -0.97 & 0.627 & 0.815 & $-3.23 ; 1.29$ \\
\hline & 2.37 & -0.89 & 0.726 & 1.051 & $-4.07 ; 2.30$ \\
\hline \multicolumn{6}{|c|}{ Suspension $(0.09 \% \mathrm{w} / \mathrm{w})$} \\
\hline \multirow{5}{*}{ UV } & 0.0774 & 3.04 & 0.752 & 4.479 & $-19.83 ; 25.91$ \\
\hline & 0.0865 & 1.40 & 0.411 & 1.376 & $-5.47 ; 8.28$ \\
\hline & 0.0910 & 0.65 & 0.417 & 1.653 & $-7.57 ; 8.86$ \\
\hline & 0.0955 & -0.92 & 0.508 & 1.848 & $-9.95 ; 8.11$ \\
\hline & 0.1046 & -4.05 & 0.785 & 4.501 & $-25.44 ; 17.35$ \\
\hline \multirow{5}{*}{ Raman } & 0.0772 & 16.06 & 2.425 & 3.035 & $6.72 ; 25.40$ \\
\hline & 0.0862 & 4.90 & 1.437 & 1.437 & $1.40 ; 8.40$ \\
\hline & 0.0908 & 0.28 & 1.653 & 2.910 & $-10.11 ; 10.67$ \\
\hline & 0.0953 & -4.08 & 2.429 & 2.429 & $-9.49 ; 1.32$ \\
\hline & 0.1044 & -12.65 & 2.744 & 2.744 & $-18.21 ;-7.09$ \\
\hline
\end{tabular}

Table 3. In-line UV and Raman quantification methods: validation parameters per concentration level for the gel and suspension. 


\begin{tabular}{|c|c|c|c|c|c|}
\hline $\begin{array}{l}\text { Spectroscopic } \\
\text { technique }\end{array}$ & $\begin{array}{l}\text { Concentration } \\
\text { level }(\% \mathrm{w} / \mathrm{w})\end{array}$ & $\begin{array}{l}\text { Uncertainty of } \\
\text { the bias (\% } \\
\text { w/w) }\end{array}$ & $\begin{array}{l}\text { Uncertainty (\% } \\
\mathbf{w} / \mathbf{w})\end{array}$ & $\begin{array}{c}\text { Expanded } \\
\text { uncertainty }(\% \\
w / w)\end{array}$ & $\begin{array}{c}\text { Relative } \\
\text { expanded } \\
\text { uncertainty (\%) }\end{array}$ \\
\hline \multicolumn{6}{|c|}{ Gel $(2 \% w / w)$} \\
\hline \multirow{5}{*}{ UV } & 1.75 & 0.0204 & 0.0447 & 0.0894 & 5.10 \\
\hline & 1.96 & 0.0286 & 0.0588 & 0.1177 & 6.01 \\
\hline & 2.06 & 0.0289 & 0.0584 & 0.1168 & 5.67 \\
\hline & 2.16 & 0.0356 & 0.0794 & 0.1588 & 7.34 \\
\hline & 2.37 & 0.0275 & 0.0590 & 0.1180 & 4.98 \\
\hline \multirow{5}{*}{ Raman } & 1.75 & 0.0200 & 0.0418 & 0.0837 & 4.78 \\
\hline & 1.96 & 0.0083 & 0.0252 & 0.0505 & 2.58 \\
\hline & 2.06 & 0.0065 & 0.0203 & 0.0405 & 1.97 \\
\hline & 2.16 & 0.0075 & 0.0190 & 0.0380 & 1.76 \\
\hline & 2.37 & 0.0114 & 0.0271 & 0.0543 & 2.29 \\
\hline \multicolumn{6}{|c|}{ Suspension $(0.09 \% \mathrm{w} / \mathrm{w})$} \\
\hline \multirow{5}{*}{ UV } & 0.0774 & 0.0020 & 0.0041 & 0.0082 & 10.63 \\
\hline & 0.0865 & 0.0007 & 0.0014 & 0.0028 & 3.20 \\
\hline & 0.0910 & 0.0009 & 0.0017 & 0.0035 & 3.82 \\
\hline & 0.0955 & 0.0010 & 0.0020 & 0.0040 & 4.20 \\
\hline & 0.1046 & 0.0026 & 0.0052 & 0.0104 & 9.94 \\
\hline \multirow{5}{*}{ Raman } & 0.0772 & 0.0011 & 0.0029 & 0.0059 & 7.63 \\
\hline & 0.0862 & 0.0004 & 0.0014 & 0.0027 & 3.14 \\
\hline & 0.0908 & 0.0013 & 0.0030 & 0.0059 & 6.53 \\
\hline & 0.0953 & 0.0006 & 0.0023 & 0.0046 & 4.85 \\
\hline & 0.1044 & 0.0007 & 0.0026 & 0.0052 & 4.99 \\
\hline
\end{tabular}

Table 4. In-line UV and Raman quantification methods: estimates of the measurement uncertainties on the API concentration at each concentration level per formulation. 


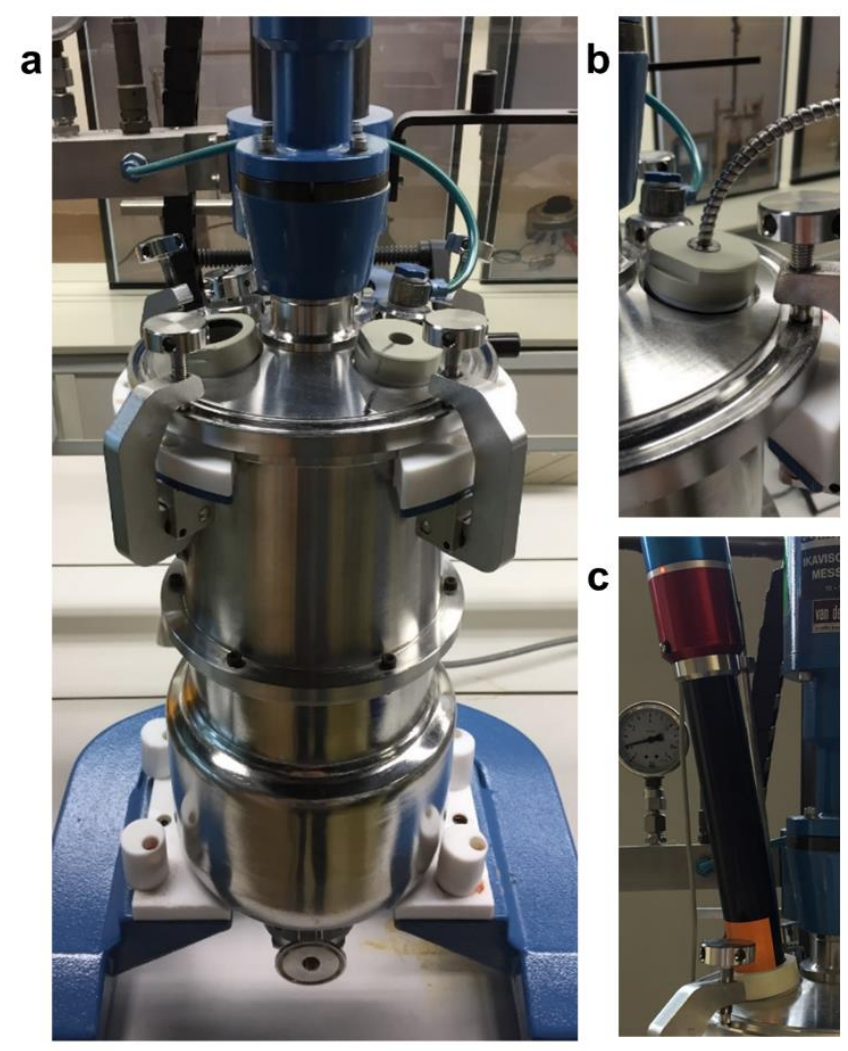

Figure 1. Experimental setup: (a) customized mixing system without probes; (b) UV immersion probe; (c) Raman PhAT probe. 


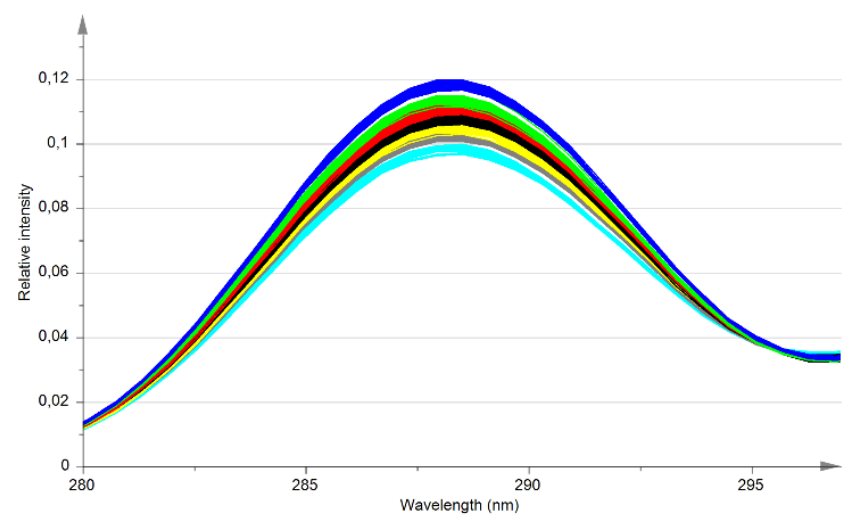

Figure 2. In-line UV spectra of the gel calibration batch between $280-300 \mathrm{~nm}$ (SNV and first derivative). Turquoise: $80 \%$, grey: $90 \%$, yellow: $95 \%$, black: $100 \%$, red: $105 \%$, green: $110 \%$, blue: $120 \%$. 


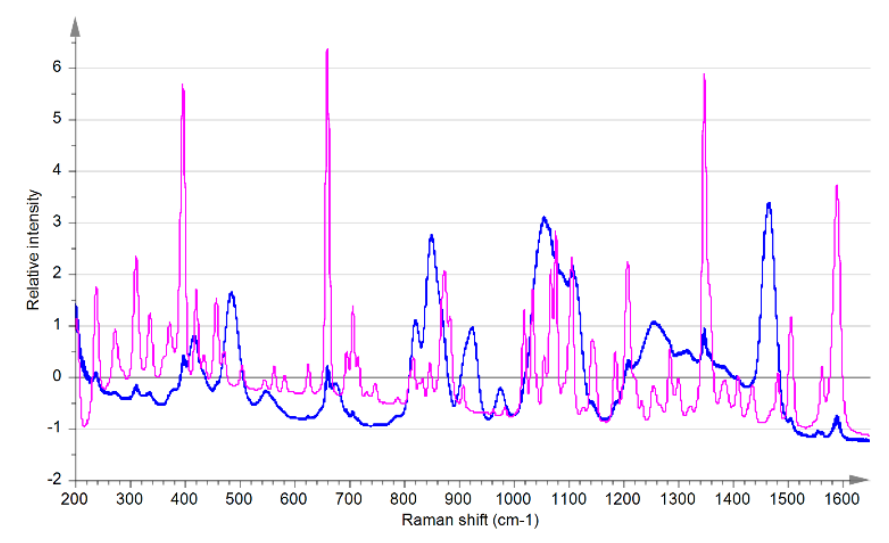

Figure 3. In-line Raman spectra (SNV) of the gel calibration batch (blue) and off-line spectra of the pure API (pink). 

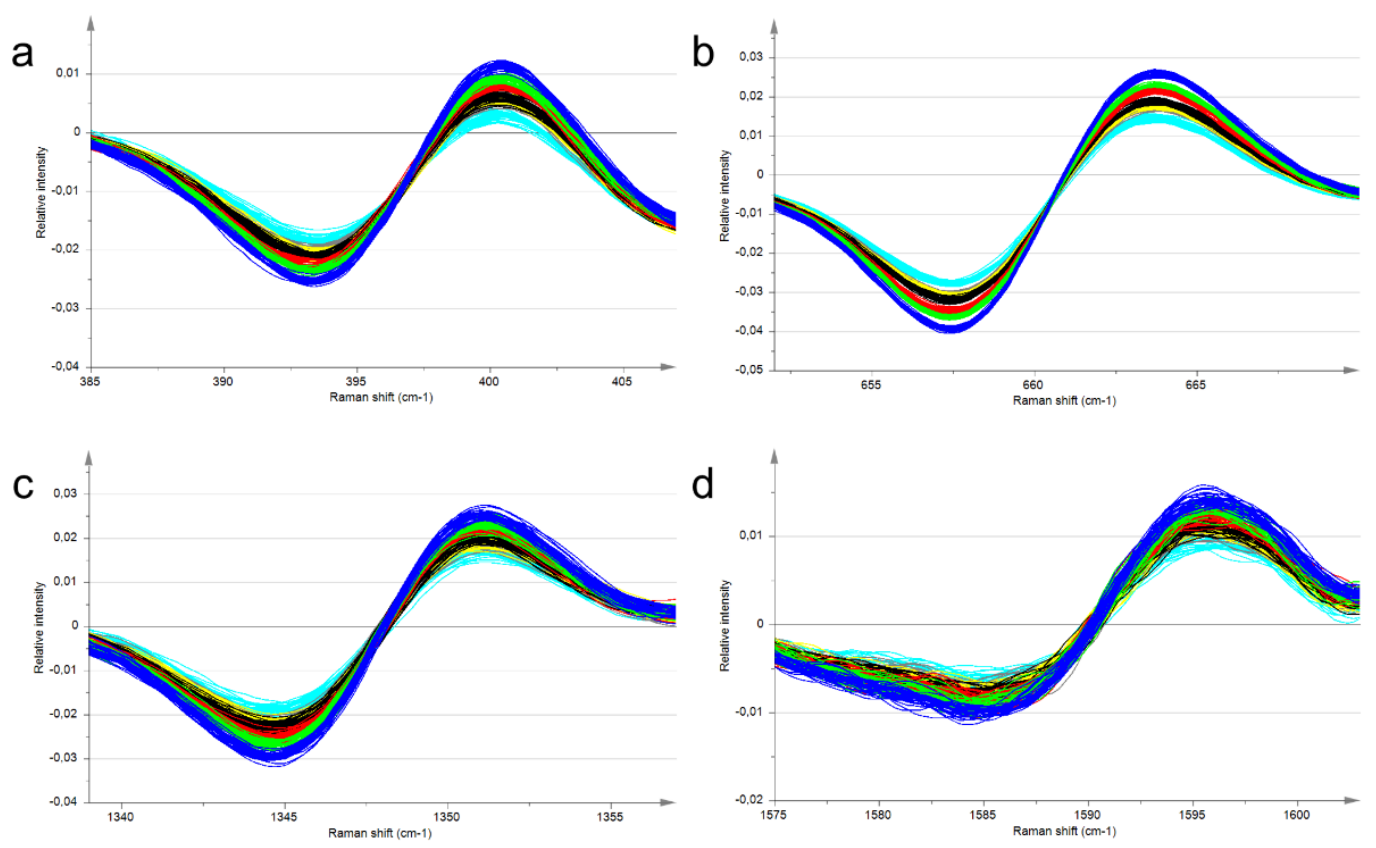

Figure 4. Detail of in-line Raman spectra (SNV and first derivative) of the gel calibration batch at the following spectral regions : (a) $385-407 \mathrm{~cm}^{-1}$, (b) $652-669 \mathrm{~cm}^{-1}$, (c) 1339 $-1357 \mathrm{~cm}^{-1}$ and (d) $1575-1602 \mathrm{~cm}^{-1}$. Turquoise: $80 \%$, grey: $90 \%$, yellow: $95 \%$, black: $100 \%$, red: $105 \%$, green: $110 \%$, blue: $120 \%$. 

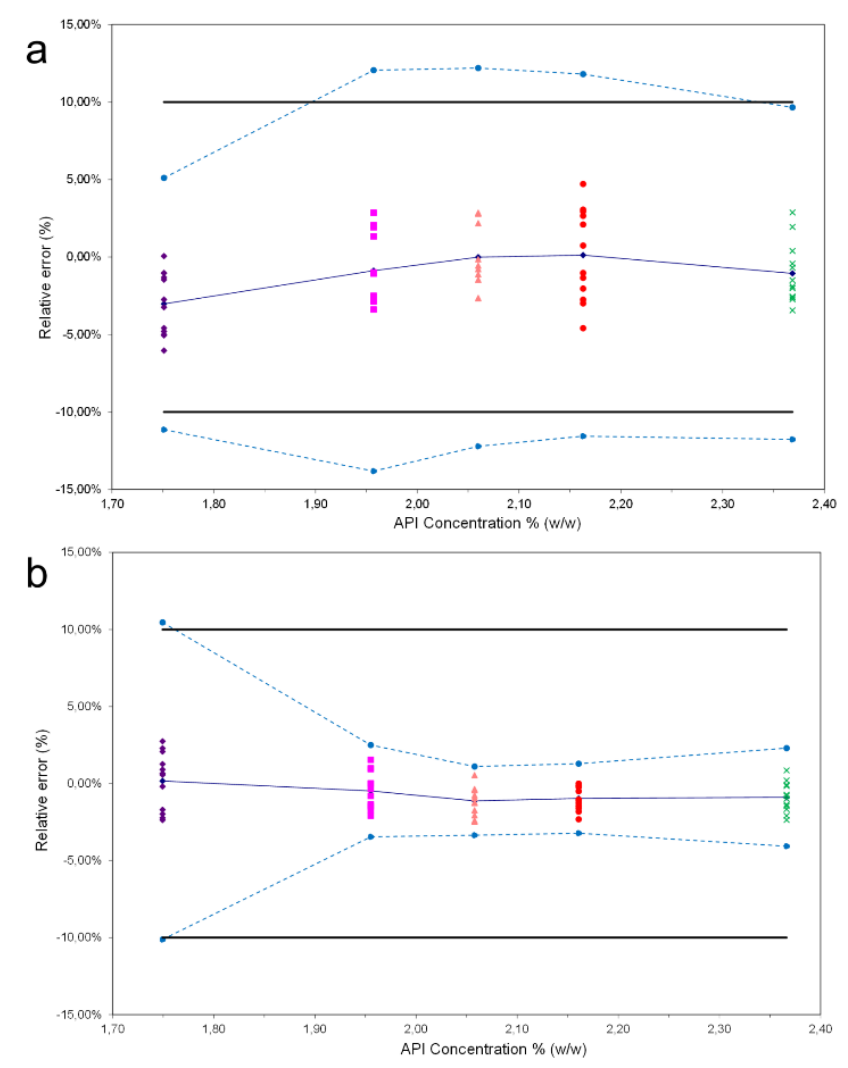

Figure 5. Accuracy profiles of the (a) UV and (b) Raman in-line quantification methods for the gel. Plain black lines: acceptance limits set at $10 \%$ (relative error), dashed blue lines: $\beta$-expectation tolerance limits, plain blue line: relative bias. 

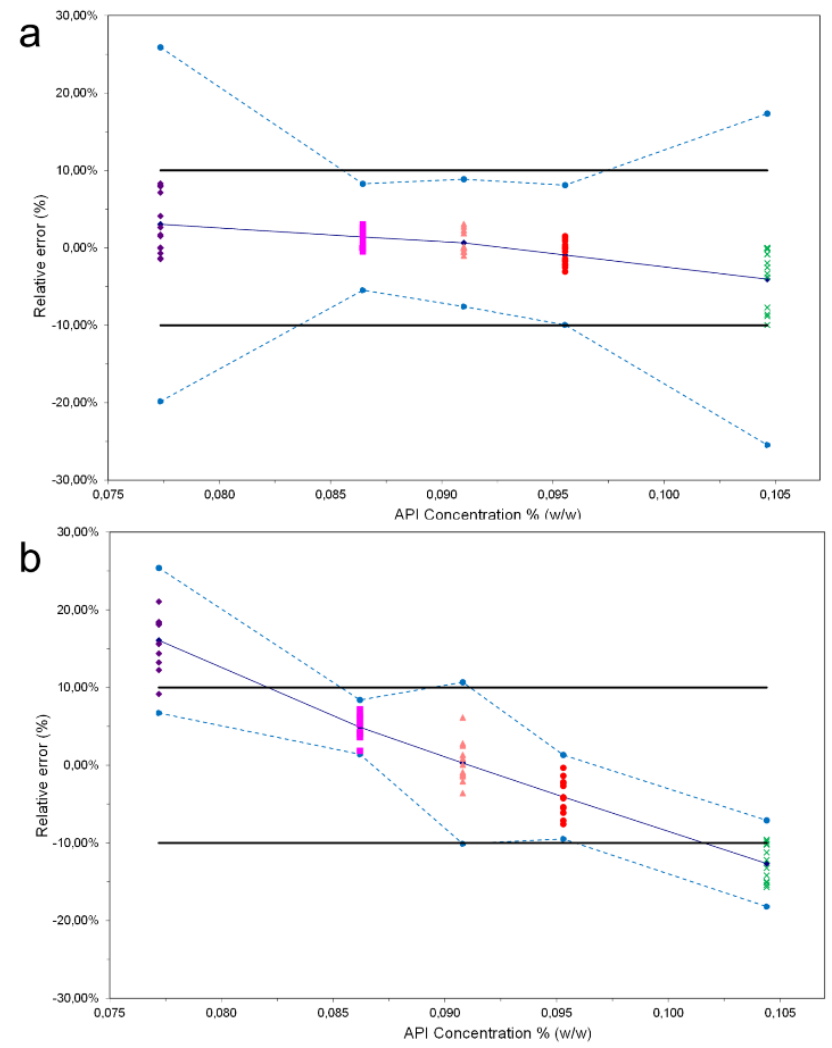

Figure 6. Accuracy profiles of the (a) UV and (b) Raman in-line quantification methods for the suspension. Plain black lines: acceptance limits set at $10 \%$ (relative error), dashed blue lines: $\beta$-expectation tolerance limits, plain blue line: relative bias. 

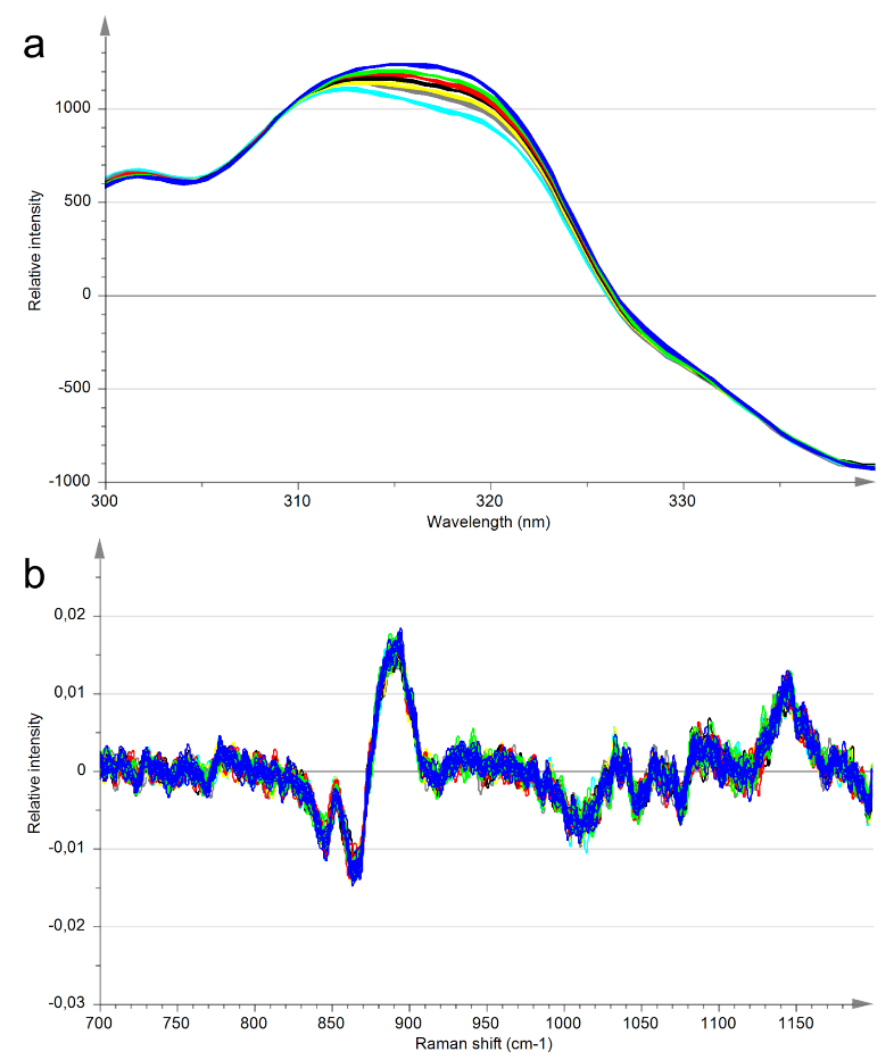

Figure 7. Preprocessed in-line (a) UV and (b) Raman spectra of the suspension calibration batch. Turquoise: $80 \%$, grey: $90 \%$, yellow: $95 \%$, black: $100 \%$, red: $105 \%$, green: 110 $\%$, blue: $120 \%$. 